\title{
Estudo do Copolímero Pirrol /Difenilamina por Espectroscopia Vibracional e Condutividade Eletrônica
}

\author{
Study of the Pyrrol/Diphenylamine Copolymer by FT-IR
}

Spectroscopy and Conductivity

\author{
Fabiele C. Dias ${ }^{1}$; Carlos A. Perez ${ }^{2}$; Henrique de Santana ${ }^{2}$
}

Resumo

O objetivo deste estudo foi analisar as propriedades físicas do copolímero formado pela deposição eletroquímica do homopolímero da polidifenilamina (PDFA) sobre o polipirrol (Ppy), e do polipirrol sobre a polidifenilamina, em diferentes condições, por meio da caracterização dos materiais formados pelas técnicas Raman ressonante, FT-IR e condutividade. Foram verificadas as interações entre as espécies presentes na nova estrutura dos copolímeros e as mudanças na condutividade eletrônica. Foi também sintetizado eletroquimicamente o copolímero PDFA/Ppy na presença do íon iodeto e o material foi caracterizado por FT-IR e condutividade. Neste estudo foi considerado o papel significativo do dopante no processo de transferência de carga entre o copolímero-dopante, atuando na estabilização das espécies na matriz polimérica e aumento da condutividade eletrônica.

Palavras-chave: Polidifenilamina. Polipirrol. Copolímero. Polímero condutor.

\section{Abstract}

The main goal of this study was to analyze the physical properties of the copolymer formed by the electrochemical deposition of the polydiphenylamine (PDPA) on polypyrrole (Ppy) and Ppy on PDPA, in different conditions, through the characterization of the materials formed by the resonant Raman, FT-IR and conductivity techniques. The interactions among the species which are present in the new copolymer structure and the changes in electronic conductivity, were verified. The copolymer was also synthesized electrochemically in the presence of iodide species and the material was characterized by FT-IR spectroscopy and conductivity. The role of the dopant was studied in the process of charge transfer between the copolymer-dopant, acting in the stabilization of the species in the polymer backbone and the increase of the electronic conductivity.

Key words: Polydiphenylamine. Polypyrrole. Copolymer. Conducting polymer.

\section{Introdução}

Devido à grande aplicação de polímeros em substituição a outros materiais, um grande esforço tem sido feito na síntese de copolímeros condutores com propriedades específicas melhoradas em relação aos homopolímeros originais.

A polidifenilamina, sintetizada em diferentes condições eletroquímicas, foi caracterizada pelas

\footnotetext{
${ }^{1}$ Bolsista CPG-UEL.

${ }^{2}$ Departamento de Química - CCE - Universidade Estadual de Londrina

Apoio: CNPq/ CPG-UEL/Fundação Araucária.
} 
espectroscopias Raman ressonante, infravermelho e reflectância (SANTANA; TEMPERINI, 1998). A copolimerização eletroquímica da difenilamina com ácido antranílico foi confirmada recentemente por FT-IR, onde Wu, Wen e Gopalan (2001) observaram a presença de unidades de ácido antranílico na cadeia do copolímero formado.

Um dos trabalhos relevantes nessa área é o Vigmond, Ghaemmaghami e Thompson (1995), e nele se utilizada a espectroscopia Raman ressonante para o polipirrol. Recentemente, Sari e Talu (1998) estudaram a síntese de copolímeros entre pirrol e anilina, usando vários solventes e eletrólitos suporte. Os autores observaram que o meio e a ordem de preparação utilizados nas combinações afetavam a estrutura e a condutividade dos materiais resultantes.

Este estudo teve como objetivo a síntese eletroquímica do copolímero formado entre pirrol e difenilamina em diferentes condições e a caracterização do produto formado pelas técnicas Raman ressonante e FT-IR. Foram levadas em consideração as propriedades dos homopolímeros e feita uma análise da mudança destas propriedades em relação ao copolímero sintetizado. Foram verificadas interações entre as espécies presentes na nova estrutura, e variações na condutividade eletrônica. Foi também sintetizado eletroquimicamente o copolímero PDFA/Ppy na presença de dopantes, tal como íon iodeto, e o material foi caracterizado por FT-IR. Neste estudo, foi considerando o papel significativo do dopante no processo de transferência de carga entre o copolímerodopante, atuando na estabilização das espécies na matriz polimérica (SANTANA; DIAS, 2003). Observou-se um aumento na condutividade eletrônica em relação ao copolímero formado utilizando outros dopantes.

\section{Materiais e Métodos}

\section{Reagentes e soluções}

Em todos os experimentos foram utilizados reagentes P. A. e a difenilamina foi previamente recristalizada duas vezes com éter de petróleo.

\section{Equipamentos}

Potenciostato Microquímica modelo MQPG - 01 acoplado ao microcomputador;

Espectrofotômetro FT-IR da Shimadzu, resolução $4 \mathrm{~cm}^{-1}$;

Espectrômetro Raman da Renishaw (Imaging Microscope System 3000). Lasers de Ar e He-Ne (Spectra Physics model 127) foram usados na radiação excitante 514,5 e $632,8 \mathrm{~nm}$, respectivamente. A resolução espectral foi de $7 \mathrm{~cm}^{-1}$.

\section{Preparação dos filmes poliméricos}

Os filmes dos copolímeros entre difenilamina (DFA) e pirrol (Py) foram preparados eletroquimicamente, sendo realizadas as sínteses em cela de um compartimento com capacidade de 10 $\mathrm{mL}$. Foi utilizado como eletrodo de trabalho um vidro condutor de $\mathrm{SnO}_{2}$ dopado com índio (ITO) e como eletrodos de referência e auxiliar, os de $\mathrm{Ag} / \mathrm{AgCl}$ e platina, respectivamente. Como eletrólito de suporte foi utilizado $\mathrm{Et}_{4} \mathrm{NBF}_{4} /$ Acetonitrila $(\mathrm{ACN})$ na síntese da PDFA e $\mathrm{HClO}_{4}$ na do PPy.

As soluções para a síntese foram preparadas previamente aos experimentos:

- Para a síntese do polipirrol (PPy): solução de 0,1 mol L-1 de Pirrol + 0,1 mol L-1 de $\mathrm{HClO}_{4}$.

- Para a síntese da polidifenilamina (PDFA): solução de $0,1 \mathrm{~mol} \mathrm{~L}^{-1}$ de DFA $+0,1 \mathrm{~mol} \mathrm{~L}^{-1}$ de $\mathrm{Et}_{4} \mathrm{NBF}_{4}$ em acetonitrila.

Para o primeiro copolímero (PPy/PDFA), foi sintetizado o filme aplicando o potencial de $1,50 \mathrm{~V}$ durante $900 \mathrm{~s}$. Após a síntese, formou-se sobre este filme o polímero da PDFA, tendo sido efetuados 100 ciclos de varredura durante a síntese, com potenciais ajustados entre $0,40 \mathrm{~V}$ a $1,40 \mathrm{~V}$.

Para o segundo copolímero (PDFA/PPY), foi sintetizado um filme de PDFA realizando 50 ciclos, nos mesmos potenciais acima citados e após a síntese formamos sobre esse filme o polímero do polipirrol com potencial controlado de $1,50 \mathrm{~V}$ durante $3600 \mathrm{~s}$. 
Os filmes foram modificados eletroquimicamente em uma solução de 0,1 mol.L ${ }^{-1}$ de $\mathrm{KI}+0,1$ mol.L-1 $\mathrm{HClO}_{4}$, realizando uma varredura de 50 ciclos com potenciais variando entre $0,40 \mathrm{~V}$ a $1,00 \mathrm{~V}$.

Os filmes preparados sobre o eletrodo de trabalho foram secos por passagem de ar frio, retirados do eletrodo e armazenados em recipientes fechados.

\section{Caracterização}

Foram obtidos os espectros FT-IR das diferentes amostras dos polímeros, os quais foram prensados em pastilhas de $\mathrm{KBr}$.

$\mathrm{Na}$ caracterização dos polímeros pela técnica Raman, foram utilizadas amostras na forma de pó e as radiações 514,5 nm de um laser de Ar e 632,8 nm de um laser de He-Ne.

As medidas de condutividade eletrônica das diferentes amostras do copolímero foram obtidas utilizando o método de duas pontas (GRUBER et al., 1997). O sólido foi inicialmente prensado por 5 min em um pastilhador de aço. Após ter sido retirado da pastilha, ele foi colocado em um suporte que continha duas pontas de contato de cobre sobre pressão constante. A área das pontas de prova foi de $1,96 \times 10^{-3} \mathrm{~cm}^{2}$. Foram acoplados a estas pontas os conectores do Potenciostato, para a realização de medidas de $\mathrm{I}=\mathrm{f}(\mathrm{V})$. Os potenciais foram variados conforme a resistência das amostras. A espessura de cada pastilha foi medida com o auxílio de um Paquímetro. Foram construídas curvas de $\mathrm{I}=\mathrm{f}(\mathrm{V})$ com o auxílio do programa Origin 5.0 e obtido o coeficiente angular desta curva. Com esses dados, foram obtidos os valores de condutividade eletrônica.

\section{Resultados e Discussão}

Foi inicialmente realizado um estudo Raman ressonante do copolímero pirrol - difenilamina, com base nas atribuições das bandas Raman características das espécies radical cátion e dicátion dos homopolímeros da difenilamina (SANTANA;
TEMPERINI, 1996; SANTANA; MATOS; TEMPERINI, 1998) e do pirrol, afim de se verificar o comportamento dessas espécies no material formado.

A radiação excitante $514,5 \mathrm{~nm}$ coloca em ressonância somente os segmentos radicais cátions no copolímero. Em observações anteriores (SANTANA, 1995), verificou-se que o espectro Raman ressonante da polidifenilamina preparada em meio neutro e após colocada em meio ácido, apresentam as mesmas freqüências atribuídas ao radical cátion da difenilbenzidina $\left(\mathrm{DFB}^{+}\right)$. Na Figura 1, foi observada uma diminuição de intensidade relativa e deslocamento da banda em $1321 \mathrm{~cm}^{-1}$ no homopolímero da difenilamina para $1326 \mathrm{~cm}^{-1}$ no espectro do copolímero. Esta banda é atribuída ao modo de estiramento $\mathrm{C}-\mathrm{C}$ entre-anéis da espécie radical cátion (SANTANA; TEMPERINI, 1996; SANTANA; MATOS; TEMPERINI, 1998; SANTANA, 1995 ). Foi observada também a diminuição de intensidade relativa da banda em 1204 $\mathrm{cm}^{-1}$, atribuída ao modo de deformação C-H no plano da espécie $\mathrm{DFB}^{+}$. A banda centrada em $1591 \mathrm{~cm}^{-1}$ no homopolímero de pirrol deslocou para $1583 \mathrm{~cm}^{-1}$ no espectro do copolímero. Esta banda é atribuída ao modo de estiramento $\mathrm{C}=\mathrm{C}$ da espécie radical cátion do pirrol $\left(\mathrm{PP}^{+}\right.$) (VIGMOND; GHAEMMAGHAMI, THOMPSON, 1995).

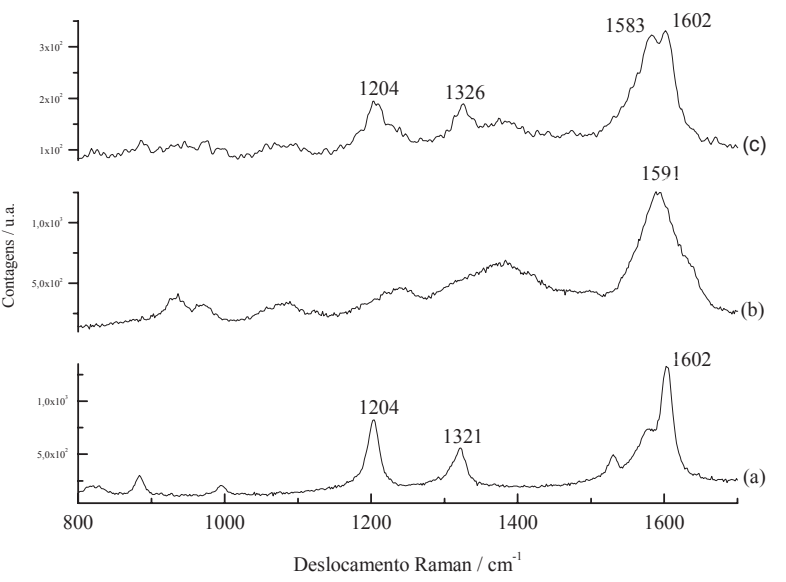

Figura 1. Espectros Raman ressonante das amostras de (a) PDFA preparada em meio neutro; (b) PPy preparada em meio ácido; (c) copolímero do PPy/ PDFA, obtidos na radiação $514,5 \mathrm{~nm}$. 
A radiação 632,8 nm possibilita observar as bandas das espécies radicais cátion e dicátion do copolímero. Na Figura 2, foram observados os deslocamentos das bandas da espécie dicátion do polipirrol em 1376, 1242 e $1087 \mathrm{~cm}^{-1}$ no homopolímero para 1370, 1227 e 1084 $\mathrm{cm}^{-1}$ no copolímero, atribuídas por Jenden, Davidson e Turner (1993) aos modos de estiramento C-N, a deformação C-H no plano anti-simétrica e simétrica, respectivamente. As bandas em 964 e $1059 \mathrm{~cm}^{-1}$, relacionadas a espécie radical cátion $\mathrm{PP}^{+}$. no homopolímero, não apresentaram variações de intensidade no espectro do copolímero. Observou-se, no copolímero, o desaparecimento da banda em 1605 $\mathrm{cm}^{-1}$, atribuída ao modo de estiramento $\mathrm{C}=\mathrm{C}$ da espécie dicátion do pirrol $\left(\mathrm{PP}^{2+}\right)$ e o aparecimento da banda em $1586 \mathrm{~cm}^{-1}$, característica da espécie radical cátion $\mathrm{PP}^{+}$. A banda em $1181 \mathrm{~cm}^{-1}$ no espectro do copolímero pode ser atribuída ao modo de deformação C-H no plano da espécie dicátion $\left(\mathrm{DFB}^{2+}\right.$ ). (SANTANA; TEMPERINI, 1996; SANTANA; MATOS; TEMPERINI, 1998; SANTANA, 1995 ).

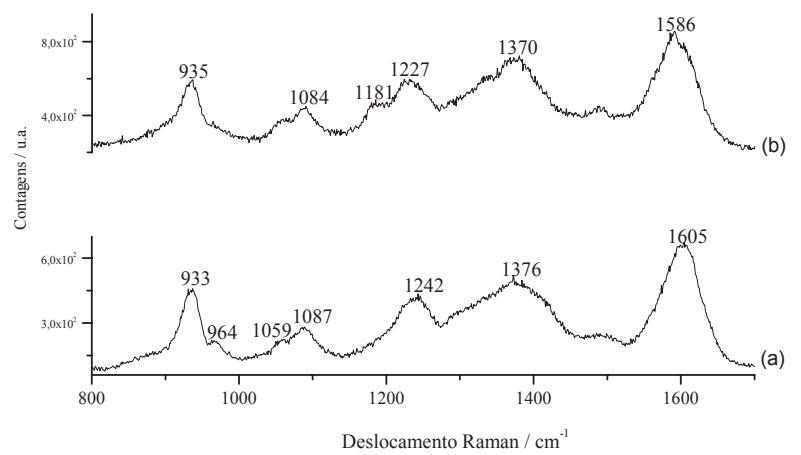

Figura 2. Espectros Raman ressonante das amostras de (a) PPy preparada em meio ácido e (b) copolímero do PPy/ PDFA na radiação $632,8 \mathrm{~nm}$.

Considerando esses resultados, podemos inferir que no copolímero ocorrem interações entre os segmentos característicos dos homopolímeros. Essa afirmativa é fundamentada no comportamento dos espectros Raman ressonante de oligômeros ou de polímeros de alto peso molecular dos homopolímeros, nos quais não se observa deslocamento de frequência.(SANTANA, 1995)
Na Figura 3, são apresentados os espectros FT-IR nas condições indicadas de polimerização. Em observações anteriores (SANTANA; DIAS, 2003) verificou-se que o espectro FT-IR da polidifenilamina preparada em meio neutro e em meio ácido apresentaram as mesmas freqüências, atribuídas ao radical cátion e dicátion da difenilbenzidina. No espectro da PDFA preparada em meio neutro (a), são observadas as freqüências 1444 e $1498 \mathrm{~cm}^{-1}$ características da espécie radical cátion $\left(\mathrm{DFB}^{+}\right)$(SANTANA; TEMPERINI, 1996; SANTANA; MATOS; TEMPERINI, 1998). No espectro do PPy preparado em meio ácido (b) foram observadas as freqüências de $1460 \mathrm{~cm}^{-1}$ atribuídas ao estiramento $(\mathrm{C}=\mathrm{N})$ e a centrada em $1542 \mathrm{~cm}^{-1}$, atribuída a combinação do estiramento $(\mathrm{C}=\mathrm{C})$ e $(\mathrm{C}-\mathrm{C})$ entre-anéis (JENDEN; DAVIDSON; TURNER, 1993) No espectro do copolímero PPy/ PDFA (c), foi observado o deslocamento das freqüências em 1444 e $1498 \mathrm{~cm}^{-1}$ para 1449 e $1495 \mathrm{~cm}^{-}$ ${ }^{1}$, características da PDFA. No espectro do copolímero PDFA/PPy (d), foi observado o surgimento da banda em $1527 \mathrm{~cm}^{-1}$, provavelmente pelo desdobramento da banda em $1542 \mathrm{~cm}^{-1}$, ocorrido pela maior interação entre os segmentos dos homopolímeros.

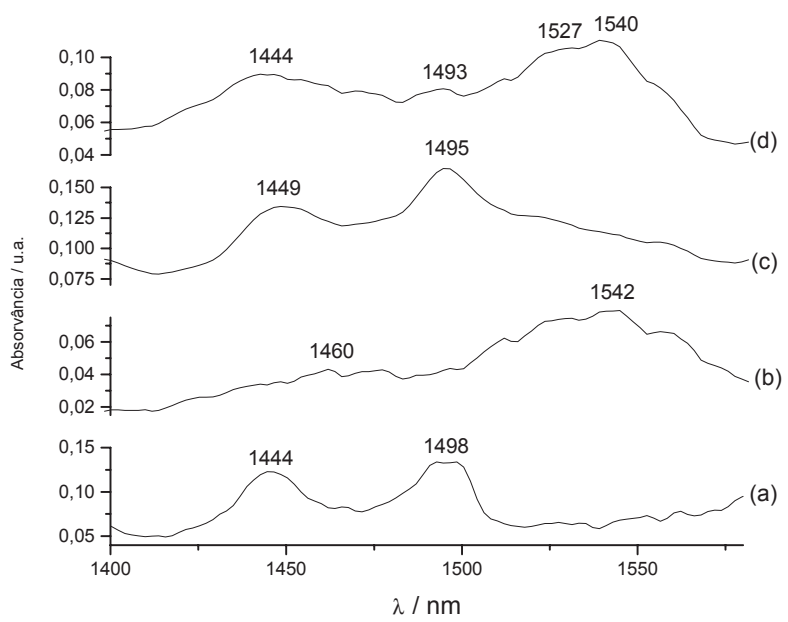

Figura 3. Espectros FT-IR da (a) PDFA preparada em meio neutro; (b) PPy preparada em meio ácido; (c) copolímero do PPy/PDFA e (d) copolímero do PDFA/PPy.

Foi verificado anteriormente (SANTANA; DIAS, 2003) que as melhores condições para estabilização das espécies radical cátion e dicátion da difenilbenzidina 
na matriz polimérica estão relacionadas com a presença dos íons de poli-iodetos $\left(\mathrm{I}_{3}^{-} \mathrm{e}_{5}^{-}\right)$como dopantes. Observou-se também aumento significativo na condutividade da polidifenilamina após este tratamento. Com o objetivo de estabilizar as espécies radical cátion e dicátion na matriz do copolímero Ppy/PDFA formado, foi realizado o mesmo processo de dopagem no copolímero.

Na Figura 4, são apresentados os espectros FT-IR nas condições indicadas de polimerização. No espectro da PDFA preparada em meio neutro com iodeto (a) foram observadas as freqüências em 1440 e $1490 \mathrm{~cm}^{-1}$. No espectro do PPy preparado em meio ácido com iodeto (b) foi observado as freqüências em $1457 \mathrm{~cm}^{-1} \mathrm{e}$ $1542 \mathrm{~cm}^{-1}$. No espectro do copolímero PPy/PDFA com iodeto (c) foi observado o deslocamento da frequência em 1440 para $1444 \mathrm{~cm}^{-1}$ e o aparecimento das freqüências em 1482 e $1496 \mathrm{~cm}^{-1}$, provavelmente pelo desdobramento da banda em $1490 \mathrm{~cm}^{-1}$, ocorrido pela maior interação entre os segmentos dos homopolímeros. No espectro do copolímero PDFA/PPy com iodeto (d), foi verificada a frequência em $1443 \mathrm{~cm}^{-1}$ (relacionada ao radical cátion da PDFA) e o surgimento de uma banda em $1529 \mathrm{~cm}^{-1}$, como foi observado anteriormente na Figura 3(d).

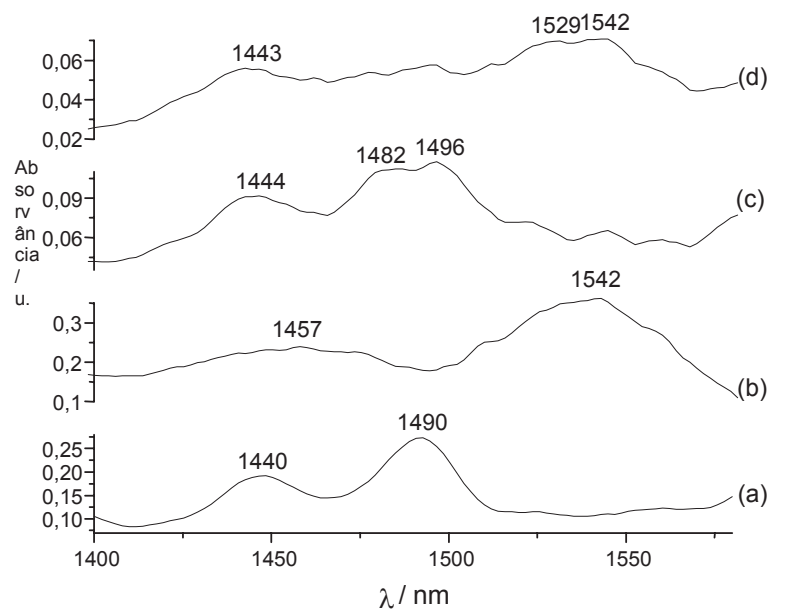

Figura 4. Espectros FT-IR da (a) PDFA preparada em meio neutro e modificada em 0,1 mol.L ${ }^{-1} \mathrm{KI}-\mathrm{HClO}_{4}$; (b) PPy preparada em meio ácido e modificado em 0,1 mol.L${ }^{1} \mathrm{KI}_{-} \mathrm{HClO}_{4}$; (c) copolímero do PPy/PDFA modificado em 0,1 mol.L-1 $\mathrm{KI}^{-\mathrm{HClO}_{4}}$ e (d) copolímero do PDFA/ PPy modificado em 0,1 mol.L-1 $\mathrm{KI}-\mathrm{HClO}_{4}$.
Com o objetivo de verificar as propriedades eletrônicas dos materiais formados nos copolímeros, foram realizadas as medidas de condutividade eletrônica, conforme Gruber et al. (1997). Na Tabela 1 e 2 são apresentados os valores de condutividade eletrônica dos homopolímeros e dos copolímeros formados, levando em consideração as diferentes condições durante a síntese eletroquímica.

Tabela 1. Medidas de Condutividade eletrônica dos materiais poliméricos formados em diferentes condições por síntese eletroquímica

\begin{tabular}{l|c}
\hline \multicolumn{1}{c|}{ CONDIÇÕES DE SÍNTESE } & $\mathbf{k}\left(\mathbf{S . c m}{ }^{-1}\right)$ \\
\hline PDFA - meio neutro $\mathrm{DFA} / \mathrm{Et}_{4} \mathrm{NBF}_{4} / \mathrm{ACN}$ & $5,2 \times 10^{-4}$ \\
\hline PPY - meio ácido Pirrol/ $/ \mathrm{HClO}_{4}$ & $1,17 \times 10^{-1}$ \\
\hline PPy/PDFA - copolímero & $3,6 \times 10^{-3}$ \\
\hline PDFA/PPY - copolímero & $5,69 \times 10^{-2}$ \\
\hline
\end{tabular}

Tabela 2. Medidas de Condutividade Eletrônica dos materiais poliméricos formados em diferentes condições por síntese eletroquímica

\begin{tabular}{|c|c|}
\hline CONDIÇÕES DE SÍNTESE & $\mathbf{k}\left({\left.\mathrm{S} . \mathrm{cm}^{-1}\right)}^{-1}\right.$ \\
\hline $\begin{array}{l}\text { PDFA - meio neutro, modificada com iodeto } \\
\text { DFA } / \mathrm{Et}_{4} \mathrm{NBF}_{4} / \mathrm{ACN}+\mathrm{KI}-\mathrm{HClO}_{4}\end{array}$ & $8,4 \times 10^{-3}$ \\
\hline PPy - meio ácido $\quad$ Pirrol $/ \mathrm{HClO}_{4}+\mathrm{KI}-\mathrm{HClO}_{4}$ & - \\
\hline PPy/PDFA - copolímero, modificado $\mathrm{KI}-\mathrm{HClO}_{4}$ & $1,03 \times 10^{-1}$ \\
\hline PDFA/PPy - copolímero, modificado $\mathrm{KI}$ - $\mathrm{HClO}_{4}$ & $3,35 \times 10^{-1}$ \\
\hline
\end{tabular}

Pode ser observado, na Tabela 1, que existe para o copolímero diferentes valores de condutividade. Para explicar esse fato, verificou-se no espectro do copolímero da Figura 3(d) uma interação entre os homopolímeros, sendo o responsável pelo melhor valor observado de condutividade. Esse fato não se repete nos valores da Tabela 2, pois, neste caso, os valores de condutividade do copolímero são próximos. Dessa forma, podemos concluir que a presença de novas freqüências nos espectros FT-IR da Figura 4 (c) e (d) pode ser um indicativo que a interação entre homopolímeros é um requisito para contribuir no aumento da propriedade condutividade. 


\section{Conclusões}

Considerando os resultados apresentados, constatou-se que as espécies dos homopolímeros se associam para formar o copolímero, essa interação entre os homopolímeros e o surgimento de novas freqüências, verificados nos espectros FT-IR é requisito para a contribuição em um melhor valor para a condutividade

O aumento da condutividade do material também está relacionado à dopagem com íons de iodeto, contribuindo na estabilização das espécies e que, como já verificado, mostra-se como melhor dopante em relação ao meio neutro e ácido.

\section{Referências}

GRUBER, J.; SANTANA, H.; GRUBER, I.; GAZOTTI, W. JR. Easily built low-cost apparatus to measure polymer conductivity. Journal of Chemical Education, Washington, v.4, p.74, 1997.

JENDEN, C. M.; DAVIDSON, R. G.; TURNER, T. G. A Fourier transform-Raman spectroscopic study of electrically conducting polypyrrole films. Polymer, Oxford, v.34, p.1649, 1993.
SANTANA, H.; DIAS, F. C. Characterization and properties of Polydiphenylamine Electrochemically modified by iodide species, Mater. Chemical Physics, Amsterdam, v.82, p.882, 2003.

SANTANA, H. Relação entre estrutura e propriedade condutora na Polidifenilamina (PDFA). Tese (Doutorado) - Universidade de São Paulo, São Paulo, 1995.

SANTANA, H.; TEMPERINI, M. L. A. The Spectroscopic Characterization of Polydiphenylamine and one of its Oligomeric Fractions. Journal of the Brazilian Chemical Society, São Paulo, v.7, p.485, 1996.

SANTANA, H.; MATOS, J. R.; TEMPERINI, M. L. A. Characterization of Polydiphenylamine Electrochemically Synthesized by Spectroscopic and Thermal Techniques. Polymer Journal, Tokio, v.30, p.315, 1998.

SARI, B.; TALU, M. Electrochemical copolymerization of pyrrole and aniline. Synthetic. Metals, Lausanne, v.94, n.2, p.221, 1998.

VIGMOND, S. J.; GHAEMMAGHAMI, V.; THOMPSON, M. Raman and resonance-Raman spectra of polypyrrole with application to sensor-gas probe interactions. Canadian Journal of Chemistry, Ottawa, v.73, n.10, p.1711-1718, 1995.

WU, M. S.; WEN, T. C.; GOPALAN, A. Electrochemical copolymerization of diphenylamine and anthranilic acid with various feed ratios. Journal of the Electrochemical Society, Baltimore, v.74, p.58, 2002. 